\title{
Coherent PON System with High-Sensitivity Polarization-Independent Receiver and no ADC/DSP
}

\author{
F. Bottoni ${ }^{(1)}$, M. Rannello ${ }^{(1)}$, M. Artiglia ${ }^{(1)}$, M. Presi $^{(1)}$ and E. Ciaramella ${ }^{(1)}$ \\ (1) Scuola Superiore Sant'Anna, V. G. Moruzzi, 1 - 56124 - Pisa (ITALY) e.ciaramella@sssup.it
}

\begin{abstract}
A 1.25 \mathrm{~Gb} / \mathrm{s}$ ASK PON system with $-51 \mathrm{dBm}$ sensitivity (at $B E R=2 \cdot 10^{-3}$ ) is enabled by a polarization-independent coherent receiver that needs no DSP (nor $A D C$ ). The system just uses common DFBs and commercial electronic devices and has $52 \mathrm{~dB}$ dynamic range.
\end{abstract}

\section{Introduction}

Coherent WDM-PON access networks are promising candidates to achieve seamless upgrade of existing fibre plants, which are based on high-loss power splitters ${ }^{1}$. Coherent systems can provide for increased power budget and much higher channel density, but these targets must be achieved with low-cost implementation of the receiver ${ }^{2}$, i.e. without expensive photonic and/or electronic components (e.g. external cavity lasers, ADC's and complex DSP).

We recently proposed a WDM-PON coherent system based on simple and effective ASK phase diversity-receiver ${ }^{3}$, where a $3 \times 3$ symmetric coupler allows coherent detection based on a common DFB as local oscillator (LO), with no special frequency control. We later introduced the extension to polarizationindependent receiver (PI-RX), which exploits the $3^{\text {rd }}$ input of the coupler and requires intradyne operation ${ }^{4}$. These demonstrations however, used offline processing in a Real Time Oscilloscope (RTO) to demodulate the signal.

Here we demonstrate for the first time a real-time implementation of the PI-RX, and thus test it in a complete and realistic system experiment. Namely, the PI-RX is realized with commercial devices, without any specific Digital Signal Processing (DSP), thus not requiring expensive ADC's: we rather use commercial high-frequency multipliers, amplifiers and combiners. Despite the very simple implementation, we still obtain $<-50 \mathrm{dBm}$ sensitivity (at FEC level), which can sustain a very high loss of the Optical Distribution Network (ODN). This can make it a promising candidate for future PON solutions.

\section{Experimental Results}

Our experimental setup is shown in fig. 1. Here, for simplicity, the transmitter was made of common DFB ( $\lambda=1540.8 \mathrm{~nm}, 10 \mathrm{MHz}$ linewidth and $-145 \mathrm{~dB} / \mathrm{Hz}$ RIN) externally modulated by a Mach-Zehnder modulator (MZM), driven at $1.25 \mathrm{~Gb} / \mathrm{s}$ by a PRBS sequence ( $2^{31}-1$ bits long). Since we are focusing on the receiver performance, we use external modulation at the transmitter in order to isolate signal-related impairments: we already demonstrated, although with offline processing, that the RX can work with directly modulated laser (DML) with similar performance ${ }^{5}$. The ODN is emulated by a $60 \mathrm{~km}$ single-mode fibre (SMF, $13 \mathrm{~dB}$ loss) a variable optical attenuator (VOA) and a Polarization Scrambler (PS), which simulates the random variations of the signal State of Polarization (SoP).

A key block in our setup is the real-time PI-RX, whose schematics is reported on the right of Fig. 1. At the $R X$ input, the $X$ and $Y$-polarization components of the signal are separated by a PBS; one of them is rotated by $90^{\circ}$ and then they both enter with parallel SoP into a symmetrical $3 \times 3$ fused-fibre power coupler ${ }^{4}$. The LO $(-3 \mathrm{dBm})$ is injected at the $3^{\text {rd }}$ input port of the coupler, with same SoP as the above two. The LO is a common DFB with same specs as the DFB in the TX, whose wavelength is tuned

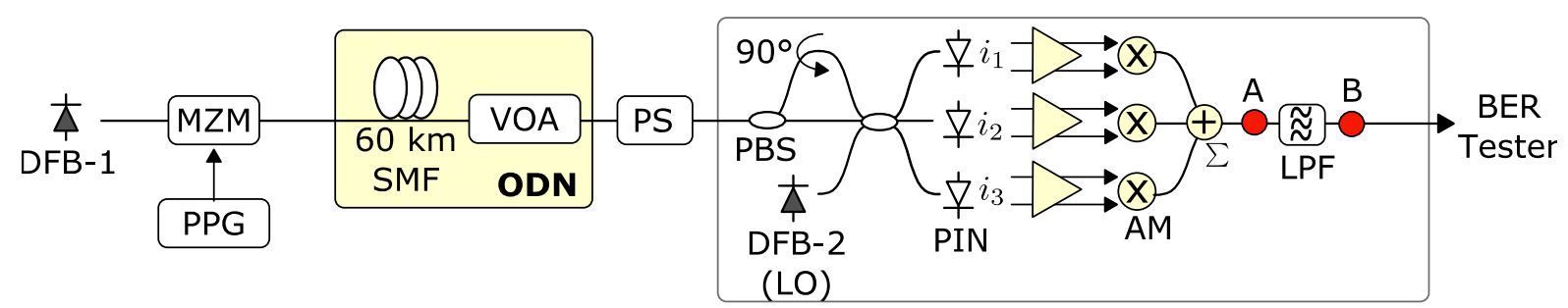

Fig. 1: Experimental Setup: DFB: Distributed FeedBack Lasers; MZM: Mach-Zehnder Modulator; SMF: Single Mode Fiber; VOA: Variable Optical Attenuator; ODN: Optical Distribution Network; PS: Polarization Scrambler; PBS: Polarization Beam Splitter; AM: Analogue Multipliers; LPF: low-pass Bessel filter (933 MHz BW); BER: Bit Error Rate Tester. Red dots: measuring points A and B. 

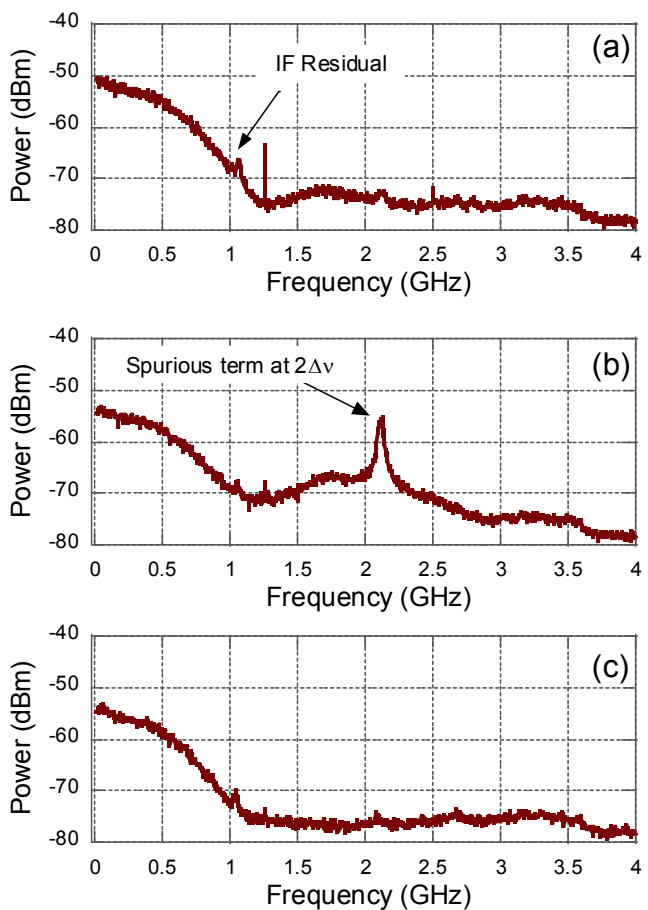

Fig. 2: Electrical signal spectra; a): taken at point A (after the adder $\Sigma$ ) when the polarization of input signal and LO are aligned; b): same as above, but with $45^{\circ}$ angle; $c$ ):

final spectrum at point $B$ (after the LPF), with $45^{\circ}$ angle.

(by thermal control) to work in intradyne regime with $\Delta v \approx 850 \mathrm{MHz} \quad(\Delta v$ : frequency difference between signal and LO). This is a condition needed to achieve polarization independence (as theoretically demonstrated ${ }^{4}$ ). The above $\Delta v$ can be varied in a $200 \mathrm{MHz}$ range without impairing the overall performance. At the three outputs, each signal is detected by a PIN+TIA photodiode (2 $\mathrm{GHz}$ bandwidth), with differential outputs. Once properly amplified by low-noise (5dB noise figure) RF amplifiers, these feed three analogue multipliers (Analog Devices ADL-5391), with $2 \mathrm{GHz}$ operating bandwidth (starting from DC). Since we feed the multipliers with the differential current pairs, the squaring operation is effectively obtained on each photocurrent. The squared signals are finally summed by an electrical power combiner $(\Sigma)$ to yield the recovered signal intensity. The sum signal is then passed through a standard lowpass filter (LPF, $4^{\text {th }}$ order Bessel, with $933 \mathrm{MHz}$ $B W)$, which is present in any NRZ receiver.

We report in Fig. 2 the electrical spectra of the recovered signal before and after the LPF. Trace (a) shows the spectrum obtained when the SoP of the input signal is aligned to the LO, which is the typical NRZ spectrum: here we also see a residual tone at $\Delta v$ frequency (in-band with the signal). This tone is due to a non-ideal behavior of the receiver components (e.g. not exact $120^{\circ}$ phase shift within the $3 \times 3$ coupler,

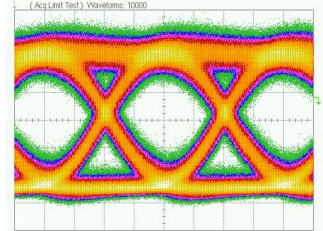

(a)

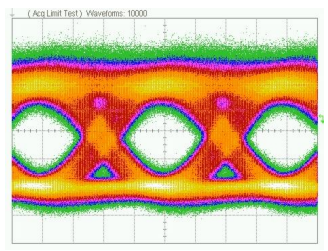

(b)
Fig. 3: Eye diagrams in B2B (taken at point B) for signal SoP aligned to LO (a) and polarization-scrambled (b). Timescale: 200 ps/div.

slightly different PIN responsivities, non-ideal frequency-response of the multipliers etc.). However, this spurious tone has very low amplitude thus it does not affect significantly the receiver performance. Trace (b) shows the spectrum taken at point $A$ in Fig. 1 when the input signal has a SoP rotated by $45^{\circ}$ in respect to the LO, which is the worst-case condition for the RX: as expected ${ }^{4}$, here a spurious tone at $2 \Delta v$ (which has maximum amplitude for this SoP) is observed. This tone has significant amplitude and might be able to impair the system. However, being at high frequency, it is effectively suppressed by the LPF, and a clear signal is indeed obtained at point $B$ in Fig. 1. The corresponding spectrum is in Fig. 2-C. As a side effect, for this input SoP, the clock line (at $1.25 \mathrm{GHz}$ ) is strongly reduced, likely due to a kind of jitter which was numerically predicted ${ }^{4}$ : however, this is not a problem for the clock and data recovery circuit.

Fig. 3 shows the eye diagrams taken after the LPF Bessel filter, when the signal SoP is exactly aligned to that of the LO and when the SoP of the signal is completely scrambled. By comparing these two eye diagrams, minor differences are observed, namely in case b) the eye diagram shows thicker traces. This has however a very limited impact on the system performance, as we see in Fig. 4, which reports the real-time bit error ratio (BER) values measured by a BER tester.

We then characterize the receiver as a function of the signal input power. In Fig. 4 we report the BER measurements of the RX. First, we show the back-to-back (B2B) measurement of the PI$\mathrm{RX}$ performance when the signal SoP is parallel to the first (black squares) or second (black triangles) axis of the PBS. In both cases, the receiver performs very well. We see that the two polarizations show a slightly different performance, likely due to a slightly different insertion loss of the PBS at its two outputs. We then stress the PI-RX by turning on the polarization scrambler (PS) just in front of it. The scrambler randomly changes (at $6 \mathrm{kHz}$ ) the signal SoP so that it uniformly covers the 


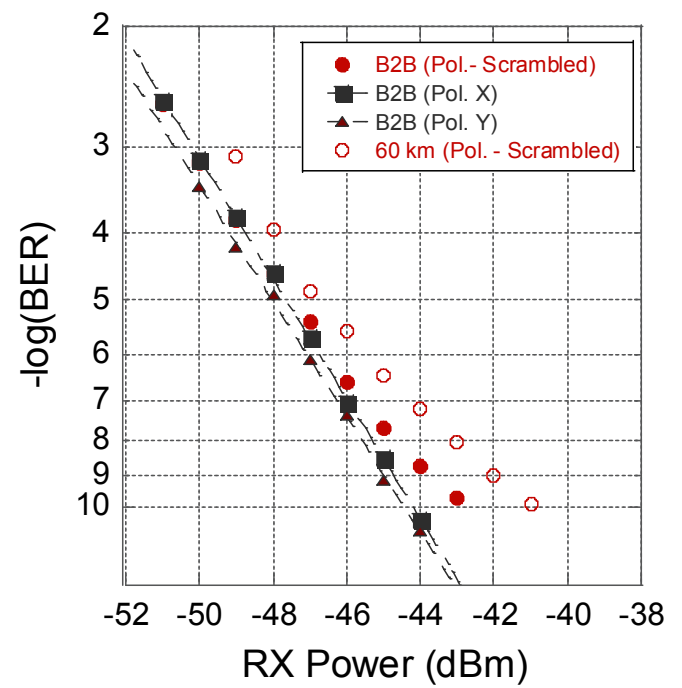

Fig. 4: Receiver Performance for different operating conditions and input signal SoP.

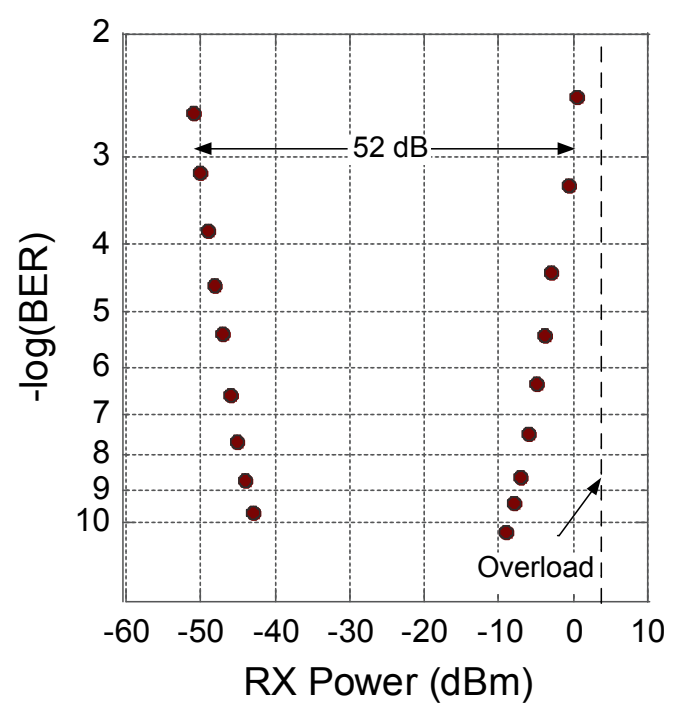

Fig. 5: Measurement of the dynamic range of the PI-RX (B2B condition).

Poincarè sphere. In this case the BER curve (red dots) shows no penalty at FEC level, whilst a penalty arises at $10^{-9}$ BER where, nevertheless, we still see the remarkable sensitivity of $-44 \mathrm{dBm}$. In any case, the sensitivity of $-51 \mathrm{dBm}$ at $10^{-3}$ BER (FEC level) corresponds to an improvement of about $10 \mathrm{~dB}$ in respect to the specs of commercially available direct-detection receivers. We note that these curves match those taken with offline processing within the experimental error $( \pm 0.5 \mathrm{~dB})$, down to around $B E R \approx 10^{-6}$ (lowest $B E R$ value that we can reliably estimate by offline processing). After transmission (red circles), a limited penalty is observed $\left(\approx 2 \mathrm{~dB}\right.$ at BER $\left.10^{-9}\right)$, which, we believe, can be reduced by further optimization of the components.
Finally, in order to prove to suitability of the solution in a real environment we perform measurements of the dynamic range of the system. Since in a real PON the ODN loss can vary largely among the different ONU's and the OLT receivers, it is critical that the $R X$ can work across a wide range of different received power levels. We thus measured the BER values as function of the input power, thus simulating a variable ODN loss. Results are reported in Fig. 5 and they show that (at FEC level) around $52 \mathrm{~dB}$ dynamic range is achieved. This value is by far higher than the expected variations in practical environments (between 15 and $23 \mathrm{~dB}$ in current standardized PON systems ${ }^{6}$ ).

\section{Conclusions}

We have presented the first implementation of a $1.25 \mathrm{~Gb} / \mathrm{s}$ ASK coherent PON system that takes advantage of a polarization independent receiver based on simple analogue processing without the need of DSP (nor ADC). We obtain a very good sensitivity (around $-51 \mathrm{dBm}$ at FEC level) despite the limited complexity (the PI-RX uses a DFB as LO and no ADC's). The PI-RX has no apparent error floor down to $B E R=10^{-10}$. No significant penalty is obtained with respect to offline processing. The measured dynamic range of the receiver is found to be around $52 \mathrm{~dB}$ at $\mathrm{BER}=10^{-3}\left(35 \mathrm{~dB}\right.$ at $\left.\mathrm{BER}=10^{-9}\right)$, largely exceeding expected needs. We finally note that the receiver scheme can be further scaled to higher bit rates (e.g. 2.5 and $10 \mathrm{~Gb} / \mathrm{s}$ ), which we previously demonstrated by offline processing only.

\section{Acknowledgements}

This work has been partially supported by the FP7 European project COCONUT G.A. 318515 and by the Italian Project ROAD-NGN. Authors wish to thank A. Rafel (BT) for fruitful discussions.

\section{References}

[1] H. Rohde et al., "Coherent optical access networks," Proc. Opt. Fiber Comm. Conf., OFC 2011, paper OTuB1

[2] J. Prat, et al., "Towards ultra-dense wavelength-to-theuser: The approach of the COCONUT project," Proc. Intern. Conf. on Transp. Opt. Networks, (2013)

[3] M. Presi, et al. "All DFB-Based Coherent UDWDM PON With $6.25 \mathrm{GHz}$ Spacing and a >40 dB Power Budget," Photon. Techn. Lett., .26, .2, pp.107-110, .2014

[4] E. Ciaramella "Polarization-Independent Receivers for low-cost Coherent OOK systems", Photon. Techn. Lett.,26, 6, pp.548-551, 2014

[5] M. Presi et al., "Low Cost $6.25 \mathrm{GHz}$ UDWDM PON based on Directly Modulated DFBs", Proc. OFC, paper Th3l.1, Los Angeles (2015)

[6] ITU-T recommendations G.984.2 (GPON), G.987.2 (XGPON) 